\title{
NUTRITIONAL RISK STATUS, DIETARY INTAKE AND COGNITIVE PERFORMANCE IN OLDER ADULTS WITH MOTORIC COGNITIVE RISK SYNDROME
}

\author{
S.A. White ${ }^{1}$, N. Ward ${ }^{2}$, J. Verghese ${ }^{3,4}$, A.F. Kramer ${ }^{5,6}$, K. Grandjean da Costa ${ }^{2}$, C.K. Liu ${ }^{1,7}$, \\ C. Kowaleski ${ }^{8}$ K.F. Reid ${ }^{1}$
}

\begin{abstract}
Background: Modifiable lifestyle factors such as diet are associated with cognitive decline and dementia. Greater understanding of the nutritional intake of older adults who are at increased risk for cognitive decline may allow for the development of more effective dietary interventions to prevent or delay the onset of dementia. Objectives: The purpose of this study was to characterize the nutritional status, diet quality and individual nutritional components of older adults with motoric cognitive risk syndrome (MCR). MCR is a pre-dementia syndrome classified by slow gait speed and subjective memory impairments. Design: Cross-sectional analysis. Setting: A community-based senior center located in an urban setting. Participants: Twenty-five community-dwelling older adults with MCR aged 60-89 yrs. Measurements: Nutritional risk status was determined using the Nestle Mini Nutritional Assessment (MNA). A food frequency questionnaire was used to quantify: overall dietary quality using the Healthy Eating Index (HEI); adherence to the Mediterranean-DASH for Neurodegenerative Delay (MIND) dietary pattern; and intake of individual nutritional components shown to be protective or harmful for cognitive function in older adults. Participants completed a computerized cognitive testing battery to assess cognitive abilities. Results: More than one third $(36 \%)$ of participants were at increased risk for malnutrition. Participants at lower risk for malnutrition had better working memory $(\mathrm{r}=0.40, \mathrm{p}=0.04)$, executive functioning $(\mathrm{r}=0.44, \mathrm{p}=0.03)$, and overall cognition $(\mathrm{r}=0.44, \mathrm{p}=0.03)$. While participants generally consumed a reasonable quality diet $(\mathrm{HEI}=65.15), 48 \%$ of participants had poor adherence to a neuroprotective MIND dietary pattern. Higher intake of B-complex vitamins was associated with better task switching $(r=0.40, p \leq 0.05)$ and faster processing speeds $(r=0.39, p \leq 0.05)$. Higher vitamin $C$ intake was associated with better executive functioning $(\mathrm{r}=0.40, \mathrm{p} \leq 0.05)$. Conclusions: Our findings suggest that a significant proportion of older adults with MCR may be at increased risk for malnutrition. While the diet quality of older adults with MCR appeared to need improvement, future studies should investigate the effects of more specific nutritional interventions, including the MIND diet, on cognition in at-risk older adults.
\end{abstract}

Key words: Motoric cognitive risk syndrome, nutritional risk, dietary patterns, cognition.

\section{Introduction}

More than 50 million people worldwide live with a diagnosis of dementia (1). The World Health Organization (WHO) estimates that the prevalence of dementia will triple by the year $2050(1,2)$. Certain modifiable lifestyle factors, including dietary intake, may delay or prevent cognitive decline and dementia later in life $(3,4)$. Further evaluation of the nutritional

1. Nutrition, Exercise Physiology and Sarcopenia Laboratory, Jean Mayer USDA Human, Nutrition Research on Aging at Tufts University, Boston, MA, USA; 2. Tufts University Department of Psychology, Medford, MA, USA; 3. Department of Neurology, Albert Einstein College of Medicine, Bronx, NY, USA; 4. Institute of Aging Research, Department of Medicine, Albert Einstein College of Medicine, Bronx, NY, USA; 5. Department of Psychology, Northeastern University, Boston, MA, USA; 6. Beckman Institute, University of Illinois, Urbana, Illinois, USA; 7. Stanford University School of Medicine, Stanford, CA, USA; 8. City of Somerville Council on Aging, Health and Human Services Department, Somerville, MA, USA

Corresponding Author: Kieran F. Reid, PhD, MPH. Nutrition, Exercise Physiology and Sarcopenia Laboratory, Jean Mayer USDA Human Nutrition Research Center on Aging at Tufts University, Boston, MA, 02111. Telephone: +1-617-556-3081; Fax: +1-617-556-3083; E-mail: kieran.reid@tufts.edu

Received September 17, 2020

Accepted for publication October 19, 2020 status of individuals at increased risk for dementia is needed to better understand how to develop and tailor more effective dietary interventions in these uniquely vulnerable older adults.

Motoric Cognitive Risk Syndrome (MCR) is a recently defined pre-dementia syndrome characterized by slow walking speed and subjective memory complaints (5-7). Individuals with $\mathrm{MCR}$ are at a substantially greater risk for the development of dementia $(6,7)$. It is predicted that $10 \%$ of the older population is living with MCR, many of whom are still community dwelling (6). Because MCR is easy to identify, quantifying the nutritional status and dietary intake of this at-risk population may lead to more effective intervention strategies for dementia prevention or risk reduction.

Studies show that older adults who have problems with memory and cognition are more likely to be at risk for malnutrition or be malnourished (8-10). Overall diet quality may independently, or in conjunction with other lifestyle factors, reduce the incidence of cognitive decline (8). Individuals who consume adequate intakes 
of vitamin E, vitamin C, caffeine, flavonoids, B vitamins, and unsaturated fatty acids are more likely to experience better cognitive outcomes later in life $(11,14,15)$. Diets high in saturated fatty acids, total fat, total calories and low in vitamin $\mathrm{D}$ have been linked to higher frequencies of cognitive impairment (11-15). However, to date, no study has quantified nutritional status, diet quality or the potential relationships between individual nutritional components and cognition in older adults with MCR.

The purpose of this study was, for the first time, to characterize nutritional risk status and overall diet quality in community-based older adults with MCR. We hypothesized that individuals with MCR would be at increased risk of malnutrition and have low adherence to healthy and neuroprotective dietary patterns. In addition, we explored the relationships between individual nutritional components and cognition in this at-risk population.

\section{Methods}

\section{Study Design}

This study utilized baseline data from a randomized controlled trial, (clinicaltrials.gov identifier: NCT03750682), which examined the real-world effects of a community-based physical activity intervention in older adults with MCR. All data were collected at a large community-based urban senior center in Greater Boston, MA, USA. The senior center provides transportation and access to wellness activities, socialization opportunities, education programs, and meal programs and serves over 5,500 community-dwelling older adults.

\section{Determination of MCR and Study Recruitment}

Participants were considered to have MCR if they met all of the following criteria: 1 . Self-reported memory complaint as assessed by Question 10 on the Geriatric Depression Scale Short Form (GDS) "Do you feel you have more problems with memory than most?" 2. Objectively defined slow gait, defined as gait speed below previously described age- appropriate mean values (age 60-74 yrs: $<0.70$ meters per second and age 75+: < 0.60 meters per second) $(6,7)$. 3 . Absence of mobilitydisability (inability to ambulate even with assistance or walking aids) 4 . Absence of dementia diagnosis. The recruitment of the study participants resulted from targeted community outreach conducted by the study investigators in close collaboration with the senior center's Health and Wellness Coordinator, as well as targeted social media advertisements. Participants who were interested in the study were pre-screened via telephone or in person and were considered eligible for an in-person screening visit if they were 60-89 years, community-dwelling (including senior housing and assisted living facilities), sedentary (not performing any structured physical activity within the past 6 months), ambulatory, and reported a subjective memory complaint. Eligible participants were then invited to participate in additional MCR screening procedures. Individuals completed a medical history questionnaire and an objective 4-meter assessment of gait speed as part of the short physical performance battery (SPPB) test (16). Participants were excluded if they had an acute or terminal illness, significant cognitive impairment (Modified Mini-Mental State Examination Score $(3 \mathrm{MSE})<80)(17)$, myocardial infarction or upper or lower extremity fracture in the previous 6 months, symptomatic coronary artery disease, or uncontrolled hypertension $(>180 / 100 \mathrm{mmHg})$. Each participant's primary care physician (PCP) confirmed the absence of a prior diagnosis of dementia. Participants who met the study entry criteria and were given medical clearance to participate by their PCP were deemed eligible. A signed informed consent was obtained from all study participants. This study was approved by the Tufts University Health Sciences Institutional Review Board.

\section{Nutritional Status and Assessment of Diet Quality}

Nutritional risk was evaluated using the Full Nestle Mini Nutritional Assessment (MNA) and characterized as either normal nutrition status, at risk for malnutrition, or malnourished. The MNA has been used in many epidemiological studies to determine the nutritional risk status of older adults with age-related cognitive decline (8-10). The MNA is a two-part assessment tool commonly used in community dwelling older adults, outpatient settings, hospitals, and nursing homes (18). This 18-item questionnaire includes anthropometric measurements, dietary intake questions, and questions about lifestyle. The sum of the MNA score is used to determine the level of nutritional risk. A score of $>24$ suggests adequate nutritional status, 17-23.5 suggests the individual is at risk for malnutrition, and $<17$ is indicative of protein-calorie malnutrition (18).

Diet quality was assessed by reviewing compliance with the Dietary Guidelines for Americans (DGA's) using the results from the 2014 Block Food Frequency Questionnaire (FFQ) and the Healthy Eating Index 2015 (HEI-2015). The 2014 Block FFQ is a 127-item questionnaire used to quantify macro- and micronutrient intake over the past year and has been used previously in large epidemiological studies (19-21). Based on recommendations from the DGA's, the HEI is a measure of diet quality using a universal set of scoring standards, determined every five years by the United States Department of Agriculture (USDA) and the Department of Health and Human Services (HHS) (22). The HEI-2015 includes 13 components and the maximum score is 100 points (23). A HEI-2015 score of 100 indicates perfect adherence to the DGA's, while a score $>80$ indicates good 
adherence, a score 51-80 represents adherence that needs improvement, a score $\leq 50$ symbolizes poor adherence, and a score of 0 demonstrates no adherence $(24,25)$.

The Mediterranean-DASH for Neurodegenerative Delay (MIND) diet is a hybrid of the Mediterranean diet and the Dietary Approaches to Stop Hypertension (DASH) diet and emphasizes individual dietary components linked to age-related cognitive decline and dementia (26). Scored on a 15-point scale, the MIND diet includes ten healthy and five unhealthy food groups. Depending on weekly consumption, the score for each category was $0,0.5$ or 1 point. The MIND diet scoring was adapted from Morris et al. and the total MIND diet score was estimated by summing the score for each of the 15 categories (26). A MIND diet score of 6.5 or below is considered low adherence to a neuroprotective dietary pattern, while a score between 7.0 and 8.0 represents moderate adherence and a score of 8.5 or above suggests high adherence. The Block FFQ was used to estimate nutrient intakes (23). FFQ's were deemed invalid if total reported energy intake was $<600 \mathrm{kcal}$ per day or $>4,000$ and $>4,200 \mathrm{kcal}$ per day for women and men respectively, or if more than twelve food items were left blank $(27,28)$. Based on results reported, we did not need to omit any FFQ's.

After an expansive review of the literature, individual nutritional components that have protective and harmful effects on cognition were selected á priori $(13,14)$. Nutritional components shown to be protective against cognitive decline selected for this analysis include: vitamin $E$, vitamin $C$, caffeine, flavonoids, vitamin $D$, B-complex vitamins, and unsaturated fatty acids $(13,14)$. Nutritional components shown to have harmful effects on cognition selected for this analysis were saturated fatty acids and total energy intake, measured in kilocalories (14).

\section{Cognitive Testing Battery}

The cognitive testing battery was conducted on a tablet device using the mobile application BrainBaseline. BrainBaseline is a scientifically-validated research tool developed by Digital Artefacts, LLC along with cognitive psychologists, and in the current study, a testing battery of eight standard cognitive tasks was used (29). Testing was administered by a research assistant in a private room and each participant was given the opportunity to take breaks as needed. The cognitive tasks included the Digit Symbol Substitution Task (DSST), the Digit Span Task, the N-Back Task, the Speed Task, the Erikson Flanker Task, the Stroop Task, the Task Switching Task, and the Trail Making Task (30-37). These individual tasks were combined into several different composite scores as is common in aging research to increase generalizability (38). Specifically, the Digit Span Task and N-Back Task comprised a Working Memory (WM) composite measure; the Flanker Task and Stroop Task comprised an Inhibitory Control (IC) composite measure; the Task Switching Task and Trail Making Task (Trails B-A) comprised a Shifting (SH) composite measure; and the Speed Task and DSST comprised a Processing Speed (PS) composite measure. In addition, an overall Executive Function (EF) composite was created by combining the individual measures from the WM, IC, and $\mathrm{SH}$ composites. Finally, an overall cognition composite was created by combining all of the individual measures from the computerized testing battery as well as the 3MSE measure. In order to create the cognitive composite scores, z-scores were calculated for the cognitive variables. Next the Stroop, Flanker, Speed, Task Switch, and Trails were multiplied by -1 so that for all measures, larger values indicate better performance whereas smaller values indicate poorer performance.

\section{Statistical Analyses}

Statistical analyses were conducted using Stata IC version 16.0 (StataCorp LLC, College Station, TX) and SAS University Edition (SAS Institute Inc. Cary, NC). All variables were assessed for normality both visually and statistically. Pearson's product-moment and Spearman's rank correlation coefficients were used to assess the relationships between cognitive composite scores and nutritional risk status, diet quality and individual nutritional components. Statistical significance was set at $p \leq 0.05$.

\section{Results}

\section{Sample Characteristics}

Descriptive statistics for the study population are presented in Table 1 . The total sample size included 25 participants with MCR. The majority of participants were female $(80 \%)$ and predominately white $(72 \%)$. More than half of the sample obtained a college degree $(56 \%)$. Polypharmacy was common and participants reported multiple chronic medical conditions. Participants had general cognitive deficits, severe mobility impairments, and slow gait speed impairments, as evidenced by their respective 3MSE, SPPB and gait speed values. More than one third $(36 \%)$ of this sample was at risk for malnutrition and no individuals were malnourished. The mean HEI2015 score was 65.15, representing DGA adherence that needs improvement. Overall adherence to the MIND dietary pattern was generally low, as $48 \%$ of participants had poor adherence, 32\% had moderate adherence, and only $20 \%$ of participants had high adherence. Based on established dietary reference intakes, it is evident that participants had low intakes of vitamin $\mathrm{E}$ and vitamin $\mathrm{D}$ and also consumed diets high in saturated fatty acids (22). 
Table 1

Sample Characteristics, Nutritional Outcomes, and Cognitive Testing Battery Scores, $\mathrm{n}=25$

\begin{tabular}{|c|c|c|c|}
\hline Sample Characteristics & Total $($ Mean \pm SD $)$ & Minimum Value & Maximum Value \\
\hline Age, years & $74.44 \pm 6.79$ & 65 & 89 \\
\hline $\mathrm{BMI}, \mathrm{kg} / \mathrm{m}^{2}$ & $32.41 \pm 6.79$ & 19.6 & 44.3 \\
\hline Medications, $\mathrm{n}$ & $6.44 \pm 4.45$ & 0 & 19 \\
\hline Medical Conditions, $\mathrm{n}$ & $4.28 \pm 2.57$ & 0 & 10 \\
\hline Geriatric Depression Scale & $2.12 \pm 1.64$ & 1 & 7 \\
\hline SPPB Score & $4.76 \pm 1.89$ & 1 & 8 \\
\hline Gait Speed, m/s & $0.52 \pm 0.11$ & 0.19 & 0.69 \\
\hline 3MSE Score & $92.44 \pm 6.67$ & 80 & 100 \\
\hline \multicolumn{4}{|l|}{ Nutritional Outcomes } \\
\hline MNA Score & $25.76 \pm 2.88$ & 19 & 30 \\
\hline Normal Nutrition Status, $\mathrm{n}$ & 16 & - & - \\
\hline At risk for malnutrition, $\mathrm{n}$ & 9 & - & - \\
\hline Malnourished, $\mathrm{n}$ & 0 & - & - \\
\hline HEI-2015 Score & $65.15 \pm 10.97$ & 45.91 & 88.33 \\
\hline MIND Diet Score & $7.1 \pm 1.8$ & 4.5 & 10.5 \\
\hline \multicolumn{4}{|l|}{ Individual Nutritional Components } \\
\hline Vitamin E, mg & $7.47 \pm 3.76$ & 1.99 & 15.83 \\
\hline Vitamin C, mg & $82.29 \pm 51.25$ & 29.43 & 215.08 \\
\hline Vitamin D, mg & $5.29 \pm 4.21$ & 1.67 & 18.41 \\
\hline Caffeine, $\mathrm{mg}$ & $122.19 \pm 126.70$ & 0.82 & 439.9 \\
\hline Flavonoids, mg & $321.59 \pm 365.02$ & 21.12 & 1187.89 \\
\hline B-Complex, mg & $41.26 \pm 11.88$ & 22.8 & 67.95 \\
\hline Unsaturated Fatty Acids, g & $41.15 \pm 22.37$ & 7.88 & 99.15 \\
\hline Saturated Fatty Acids, g & $24.03 \pm 15.73$ & 6.30 & 69.53 \\
\hline Total Kcal & $1574 \pm 729.63$ & 619.23 & 3534.95 \\
\hline \multicolumn{4}{|l|}{ Cognitive Testing Battery Scores } \\
\hline DSST, $\mathrm{n}$ & $23.28 \pm 9.51$ & 4 & 40 \\
\hline Digit Span, $\mathrm{n}$ & $5.36 \pm 2.23$ & 0 & 10 \\
\hline N-Back 1-Back Accuracy, \% & $0.74 \pm 0.23$ & 0.37 & 1.00 \\
\hline N-Back 2-Back Accuracy, $\%$ & $0.57 \pm 0.21$ & 0.22 & 0.88 \\
\hline Speed Accuracy, $\%$ & $0.90 \pm 0.11$ & 0.47 & 1.00 \\
\hline Speed Reaction Time, ms & $447.90 \pm 114.04$ & 309.00 & 724.09 \\
\hline Flanker Effect, ms & $19.3 \pm 56.82$ & -92.47 & 201.01 \\
\hline Flanker Incongruent, $\mathrm{ms}$ & $753.81 \pm 177.45$ & 539.4 & 1183.66 \\
\hline Stroop Effect, ms & $293.85 \pm 198.56$ & -193.33 & 670.42 \\
\hline Task Switching Cost, ms & $417.23 \pm 313.88$ & -67.58 & 1123.52 \\
\hline Trails, ms & $41611.75 \pm 57464.03$ & -46758 & 158421 \\
\hline
\end{tabular}

Abbreviations: BMI, Body Mass Index; kg, kilograms; m, meters; n, number of; m/s, meters per second; 3MSE, Modified Mini Mental Status Exam; MNA, Mini Nutritional Assessment;

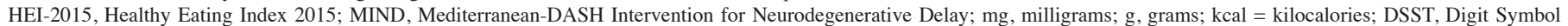
Substitution Test, one-minute version; \%, percent correct; ms, milliseconds 
Figure 1

Linear relationship between cognitive composite z-scores and risk of malnutrition
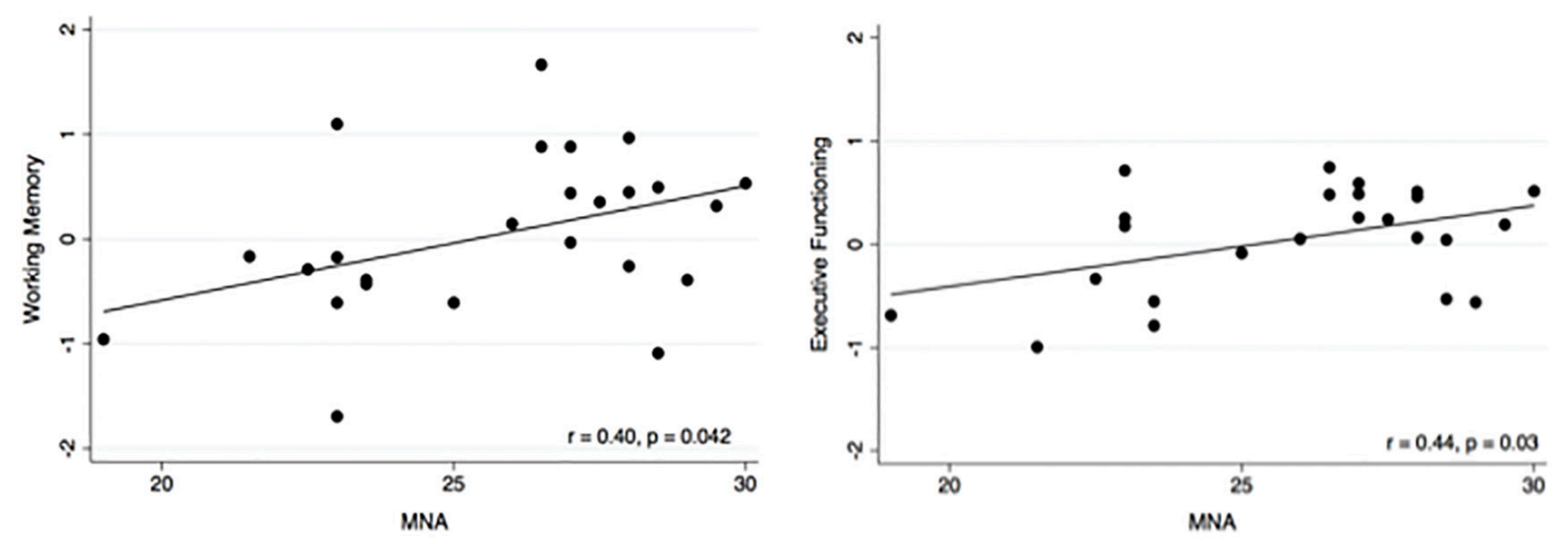

Figure 1a: Working Memory and MNA

Figure 1b: Executive Functioning and MNA

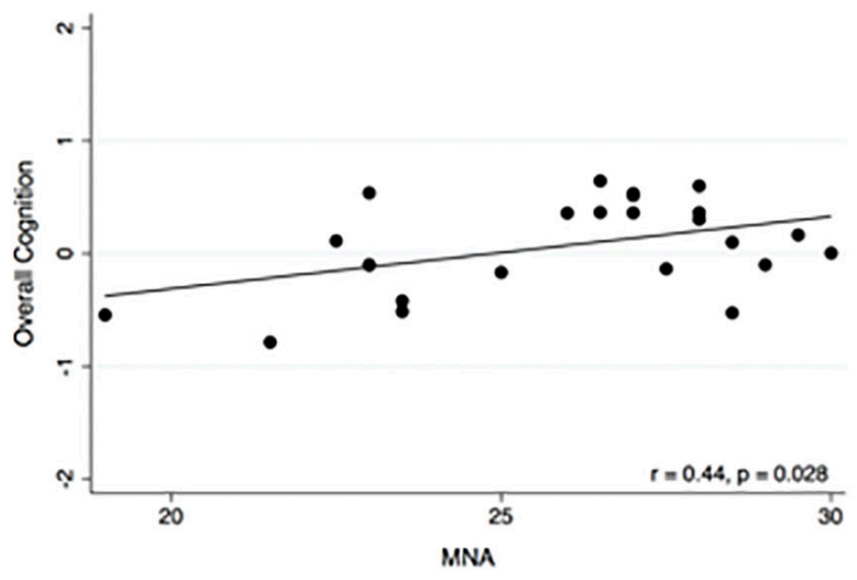

Figure 1c: Overall Cognition and the MNA

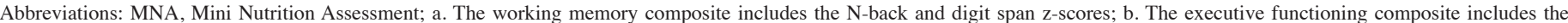

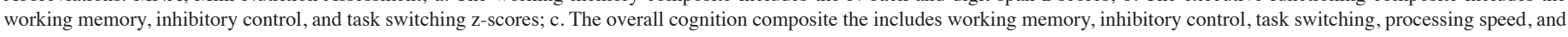
3MSE z-scores

\section{Associations Between Nutritional Status and Cognitive Composite Scores}

Figures 1a-c represents the statistically significant linear relationships between level of nutritional risk and cognitive composite scores. Participants with normal nutrition status, determined by higher scores on the MNA, performed better than participants at risk for malnutrition on tasks of working memory $(r=0.40, p$ $=0.04)$, executive functioning $(\mathrm{r}=0.44, \mathrm{p}=0.03)$, and overall cognition $(\mathrm{r}=0.44, \mathrm{p}=0.03)$.

\section{Relationships Between Individual Nutritional Components and Cognitive Composite Scores}

Table 2 is a correlation matrix of the nine individual nutritional components and cognitive composite scores included in this analysis. We observed three notable associations between individual nutrients and cognitive composite scores. Higher intake of B-complex vitamins was positively associated with better task switching $(\mathrm{r}=$ $0.40, \mathrm{p} \leq 0.05)$ and processing speed $(\mathrm{r}=0.39, \mathrm{p} \leq 0.05)$. We also found that a higher intake of vitamin $C$ was positively associated with better performance in tasks of executive functioning $(\mathrm{r}=0.40, \mathrm{p} \leq 0.05)$. These results, however, should be interpreted cautiously, as these analyses were not adjusted for multiple comparisons.

\section{Discussion}

This study is the first to examine nutritional risk status, dietary quality, and individual nutritional components in older adults with MCR. Our major findings revealed that a high proportion of older adults with MCR were 
Table 2

Correlation matrix of outcome measures

\begin{tabular}{|c|c|c|c|c|c|c|c|c|c|}
\hline & Vitamin E, mg & Vitamin C, mg & Caffeine, mg & Flavonoids, mg & Vitamin D, mcg & B-Complex, mg & Unsat. FA's g & Sat. FA, g & Total Kcal \\
\hline Processing Speed & 0.13 & 0.04 & 0.14 & 0.14 & 0.07 & $0.39 * *$ & 0.15 & 0.22 & 0.13 \\
\hline Working Memory & 0.09 & 0.15 & -0.21 & 0.03 & 0.23 & 0.29 & 0.12 & 0.02 & 0.04 \\
\hline Inhibitory Control $^{\wedge}$ & 0.12 & 0.25 & -0.14 & 0.19 & 0.12 & -0.02 & 0.19 & 0.15 & 0.18 \\
\hline Shifting^ & 0.11 & 0.22 & -0.05 & -0.05 & 0.22 & $0.40 * *$ & 0.16 & 0.04 & 0.08 \\
\hline Executive Functioning & 0.09 & $0.40^{* *}$ & -0.12 & -0.02 & 0.18 & 0.31 & 0.11 & 0.04 & 0.08 \\
\hline Overall Composite & 0.03 & 0.19 & 0.03 & -0.04 & 0.14 & 0.19 & 0.07 & 0.08 & 0.06 \\
\hline
\end{tabular}

Abbreviations: $\mathrm{mg}$, milligrams; mcg, micrograms; unsat, unsaturated; sat, saturated; g, grams; kcal, kilocalories; a. All cognition variables are composite z-scores; b. The processing speed composite includes the speed test and DSST z-scores; c. The working memory composite includes the N-Back test and digit span test z-scores; d. The inhibitory control composite includes the stroop test and flanker test z-scores; e. The shifting composite includes the task switching and trails z-scores; f. The executive functioning composite includes working memory, inhibitory control and task switching z-scores; g. The overall composite includes the working memory composite, inhibitory control composite, shifting, processing speed composite and 3MSE composite; h. ^ Spearman rank correlation coefficient; i. **p $\leq 0.05$

at risk for malnutrition. Higher scores on the MNA, which correspond to a lower risk of being malnourished, were positively associated with working memory, executive functioning and overall cognition. Based on the HEI-2015 scores, our sample consumed diets that need improvement. The MIND diet scores, however, showed that participants had low adherence to a diet recently shown to be protective against cognitive decline. Our exploratory analysis revealed that individuals with higher intakes of B-complex vitamins performed better on tests of task switching and processing speed and higher intakes of vitamin $\mathrm{C}$ was associated with better executive functioning.

The level of nutritional risk identifies severity of deficiency, impairment status, and the point at which nutritional intervention is necessary. A high percentage of participants $(36 \%)$ were at risk of becoming malnourished. This was supported by the literature, which suggests between $34 \%$ to $58 \%$ of individuals with mild cognitive impairment (MCI) and dementia are at risk of malnutrition based on the MNA (8-10). In accordance with Khater et al, we also found strong, positive relationships between the level of nutritional risk and cognitive performance (9). Participants at higher risk of malnutrition had poorer performance on working memory, executive functioning and overall cognition compared to participants with normal nutritional status. Shim et al previously found no difference in the nutritional risk status of people with and without MCR (39). This study, however, may not be comparable to our study findings, as it was conducted in the Korean population and did not use the full length, 18-item MNA, which may be a more comprehensive way to capture malnutrition in older adults.

From our dietary quality analyses, we found that participants consumed diets that need improvement and their adherence to a potentially neuroprotective dietary pattern was low. The HEI-2015 scores in our sample aligned with the USDA average of 64.0 for the general older adult population, who notably consume the highest quality diets of all other age groups (22). Similar to our findings, the CHAP Study and Ye et al. did not find any associations between the HEI and cognitive functioning $(40,41)$. Recent studies have established that adherence to the specific neuroprotective MIND dietary pattern, characterized by ten healthy and five unhealthy food groups, is associated with a substantially reduced risk for dementia in older adults (42). In our sample, $48 \%$ of participants had low adherence, while only $20 \%$ had high adherence to the MIND diet. Morris et al. concluded that individuals who had high adherence to the MIND diet may be 53\% less likely to develop Alzheimer's disease compared to those with low adherence (26). Based on our findings, more studies are needed to understand the effects of specific dietary interventions for mitigating cognitive decline and dementia.

Several nutritional components and food groups, including antioxidants, B vitamins, and unsaturated fatty acids have consistently been linked to better cognitive outcomes and the preservation of cognition later in life (11-15). We found that higher intakes of B-complex vitamins were associated with better scores on tests of task switching and processing speed. In accordance with our findings, the FACIT trial found that individuals with low B vitamin intake performed worse on tests of sensorimotor speed, processing speed, and complex switching tasks than participants with higher B vitamin intake (43). Further, B-complex vitamins play a key role in brain function, specifically with regard to regulating homocysteine, which may be a risk factor for cognitive impairment, dementia, and Alzheimer's disease (44). Adequate intakes of B vitamins may potentially lower the risk for cognitive decline and dementia in individuals with elevated homocysteine. We also found a positive association between vitamin $C$ intake and executive functioning. Similarly, Engelhart et al. found that high intakes of vitamin $\mathrm{C}$ may be associated with better cognitive functioning and a lower incidence of Alzheimer's disease (45). While studies have found that vitamin $C$ is beneficial for improving overall health in older adults, its role in improving cognition and delaying dementia is not as well established $(46,47)$. Future studies 
should explore the potential for supplementation with a focus on adequate nutrient intakes in this population, particularly with regard to vitamin $\mathrm{C}$ and the B-complex vitamins.

This study had several strengths and limitations. All study procedures were conducted at a senior center, allowing us to reach a real-world population of community dwelling older adults with MCR that would typically not be well-represented in clinical trials. However, one weakness of this study was that an FFQ was used to quantify micronutrient intake, which limits the validity and reproducibility of quantified nutrient intakes. A 24-hour food recall may have been a more appropriate measure of micronutrient intake. Additionally, the cross-sectional nature of our analyses precludes definitive causal inferences between nutritional intake and cognitive performance in this at-risk population. Further, because the senior center was an aggregate meal site that served one meal per day to the participants, the HEI-2015 scores may have been biased. It is also possible that because subjects may have had emerging cognitive and memory deficits, our results may have been specifically influenced by recall bias. Another limitation of our study was the small sample size which limits the statistical power and generalizability of our findings. Nonetheless, even with our relatively small size, we found several notable and statistically significant relationships that should be further examined in larger studies.

In conclusion, our study found that a significant proportion of older adults with MCR were at an increased risk for malnutrition. While participants reported dietary quality that needs improvement based on the HEI-2015, individuals also had low adherence to a neuroprotective dietary pattern known to substantially delay the onset on dementia. Higher intakes of B-complex vitamins and vitamin $\mathrm{C}$ are associated with better cognitive performance in MCR, although larger scale studies are needed to verify these findings. In addition, future studies should also investigate the effects of more specific nutritional interventions, such as the MIND diet, in at risk older adults.

Funding: This research was supported by the Boston Claude D. Pepper Older Americans Independence Center (1P30AG031679), the National Center for Advancing Translational Sciences, National Institutes of Health (NIH) (UL1TR001064) and is based on the work supported by the U.S. Department of Agriculture, under agreement No. 58-1950-4-003. Any opinions, findings, conclusion, or recommendations expressed in this publication are those of the author(s) and do not necessarily reflect the view of the U.S. Department of Agriculture.

Acknowledgements: We thank the following individuals for supporting this project: Joan Severson, Digital Artefacts LLC; and Joseph A. Curtatone, Mayor of Somerville, MA.

Conflicts of Interest: The authors state no conflicts of interest.

Ethical standard: The authors declare the study procedures comply with all ethical standards.

\section{References}

1. World Health Organization. Dementia. 2019. https://www.who.int/newsroom/ fact-sheets/detail/dementia. Accessed 13 April 2020

2. Minghui R. Risk reduction of cognitive decline and dementia: Who Guidelines. 2019. World Health Organization. ISBN-13: 978-92-4-155054-3. Accessed 13 April 2020

3. Zhao C, Noble JM, Marder K, Hartman JS, Gu Y, Scarmeas N. Dietary Patterns, Physical Activity, Sleep, and Risk for Dementia and Cognitive Decline. Curr Nutr Rep. 2018;7(4):335-345. doi:10.1007/s13668-018-0247-9

4. Canevelli M, Lucchini F, Quarata F, Bruno G, Cesari M. Nutrition and Dementia: Evidence for Preventive Approaches? Nutrients. 2016 Mar 4;8(3):144. doi: $10.3390 /$ nu8030144

5. Ayers E, Verghese J. Motoric cognitive risk syndrome and risk of mortality in older adults. Alzheimers Dement. 2016;12(5):556-564. doi:10.1016/j jalz.2015.08.167

6. Verghese J, Annweiler C, Ayers E, Barzilai N, Beauchet O, Bennett DA, et al. Motoric cognitive risk syndrome: multicountry prevalence and dementia risk. Neurology. 2014;83(8):718-26. doi:10.1212/WNL.0000000000000717

7. Verghese J, Wang C, Lipton RB, et al. Motoric cognitive risk syndrome and the risk of dementia. J Gerontol A Biol Sci Med Sci. 2013;68(4):412-418 doi:10.1093/gerona/gls191

8. Ogawa S. Nutritional management of older adults with cognitive decline and dementia. Geriatr Gerontol Int. 2014;14 Suppl 2:17-22. doi:10.1111/ggi.12252

9. Orsitto G, Fulvio F, Tria D, et al. Nutritional status in hospitalized elderly patients with mild cognitive impairment. Clin Nutr. 2009;28(1):100-102. doi:10.1016/j.clnu.2008.12.001

10. Khater MS, Abouelezz NF. Nutritional status in older adults with mild cognitive impairment living in elderly homes in Cairo, Egypt. J Nutr Health Aging. 2011;15(2):104-108. doi:10.1007/s12603-011-0021-9

11. Cao L, Tan L, Wang HF, et al. Dietary Patterns and Risk of Dementia: A Systematic Review and Meta-Analysis of Cohort Studies. Mol Neurobiol. 2016;53(9):6144-6154. doi:10.1007/s12035-015-9516-4

12. Torres SJ, Lautenschlager NT, Wattanapenpaiboon N, et al. Dietary pattern are associated with cognition among older people with mild cognitive impairment. Nutrients. 2012;4(11):1542-1551. doi:10.3390/nu4111542

13. van de Rest $\mathrm{O}$, Berendsen AA, Haveman-Nies A, et al. Dietary patterns, cognitive decline, and dementia: a systematic review. Adv Nutr. 2015;6(2):154-168. doi:10.3945/an.114.007617

14. Smith PJ, Blumenthal JA. Dietary Factors and Cognitive Decline. J Prev Alzheimers Dis. 2016;3(1):53-64. doi:10.14283/jpad.2015.71

15. Shishtar E, Rogers GT, Blumberg JB, R Au, PF Jacques. Long-term dietary flavonoid intake and risk of Alzheimer disease and related dementias in the Framingham Offspring Cohort. Am J Clin Nutr. 2020;112(2):343-353. doi:10.1093/ajen/nqaa079

16. Guralnik JM, Simonsick EM, Ferrucci L, et al. A short physical performance battery assessing lower extremity function: association with self-reported disability and prediction of mortality and nursing home admission. J Gerontol. 1994;49(2):M85-M94. doi:10.1093/geronj/49.2.m85

17. Teng EL, Chui HC. The Modified Mini-Mental State (3MS) examination. J Clin Psychiatry. 1987;48(8):314-318.

18. Guigoz Y, Lauque S, Vellas BJ. Identifying the elderly at risk for malnutrition. The Mini Nutritional Assessment. Clin Geriatr Med. 2002;18(4):737-757. doi:10.1016/s0749-0690(02)00059-9

19. Subar AF, Thompson FE, Kipnis V, Midthune D, Hurwitz P, McNutt S, McIntosh A, Rosenfeld S. Comparative validation of the Block, Willett, and National Cancer Institute food frequency questionnaires: the Eating at America's Table Study. Am J Epidemiol. 2001 Dec 15;154(12):1089-99. doi:10.1093/aje/154.12.1089

20. Akter S, Dawson J, Chin SH, Binks M. Associations Among Diet QualityRelated Biomarkers, Block Food Frequency Questionnaire, and 24-hour Dietary Recall. Curr Dev Nutr. 2020;4(Suppl 2):1374. doi:10.1093/cdn/ nzaa061_002

21. Ducharme-Smith K, Rosenstock S, Garcia-Larsen V, Larzelere F, Chambers R, Kenney A, et al. Quality of Diet and Cardio-Metabolic Outcomes in Native American Adolescents Participating in the Together on Diabetes Home-Visiting Program, Curr Dev Nutr. 2020 Jun;4(2):180. doi:10.1093/cdn/ nzaa043_031

22. United States Department of Agriculture. HEI Scores for Americans. 2019. https: / / www.fns.usda.gov/ hei-scores-americans. Retrieved 1 May 2020.

23. Nutrition Quest. Assessment \& Analysis Services. 2014. https: / nutritionquest com/assessment/list-of-questionnaires-and-screeners/. Accessed 26 April 2019.

24. Guenther PM, Casavale KO, Reedy J, et al. Update of the Healthy Eating Index: HEI-2010. J Acad Nutr Diet. 2013;113(4):569-580. doi:10.1016/j jand.2012.12.016

25. Al-Ibrahim AA, Jackson RT. Healthy eating index versus alternate healthy index in relation to diabetes status and health markers in U.S. adults NHANES 2007-2010. Nutr J. 2019;18(1):26. doi:10.1186/s12937-019-0450-6

26. Morris MC, Tangney CC, Wang Y, et al. MIND diet associated with reduced incidence of Alzheimer's disease. Alzheimers Dement. 2015;11(9):1007-1014 doi:10.1016/j.jalz.2014.11.009 
27. Rimm EB, Giovannucci EL, Stampfer MJ, Colditz GA, et al; Am J Epidemiol 1992;135(10):1114-26. doi.org/10.1093/oxfordjournals.aje.a116211

28. Salvini S, Hunter DJ, Sampson L, Stampfer MJ, et al. Food-Based Validation of a Dietary Questionnaire: The Effects of Week-to-Week Variation in Food Consumption. Int. J. Epidemiol. 1992;18(4):858-867. doi.org/10.1093/ ije / 18.4.858

29. Lee H, Baniqued PL, Cosman J, et al. Examining cognitive function across the lifespan using a mobile application. Computers in Human Behavior 2012;28(5): 1939-1946. doi.org/10.1016/j.chb.2012.05.013

30. Hoyer WJ, Stawski RS, Wasylyshyn C, et al. Adult age and digit symbol substitution performance: a meta-analysis. Psychol Aging. 2004;19(1):211-214. doi:10.1037/0882-7974.19.1.211

31. Conway ARA, Kane MJ, Bunting MF, et al. Working memory span tasks: A methodological review and user's guide. Psychonomic Bulletin \& Review 2005;12:769-786. doi.org/10.3758/BF03196772

32. Owen AM, McMillan KM, Laird AR, et al. N-back working memory paradigm: a meta-analysis of normative functional neuroimaging studies. Hum Brain Mapp. 2005;25(1):46-59. doi:10.1002/hbm.20131

33. Basner M, Dinges DF. Maximizing sensitivity of the psychomotor vigilance test (PVT) to sleep loss. Sleep. 2011;34(5):581-591. doi:10.1093/sleep/34.5.581

34. Fan J, McCandliss BD, Fossella J, et al. The activation of attentional networks. Neuroimage. 2005;26(2):471-479. doi:10.1016/j.neuroimage.2005.02.004

35. MacLeod CM. Half a century of research on the Stroop effect: an integrative review. Psychol Bull. 1991;109(2):163-203. doi:10.1037/0033-2909.109.2.163

36. Monsell S. Task switching. Trends Cogn Sci. 2003;7(3):134-140. doi:10.1016/ s1364-6613(03)00028-7

37. Tombaugh TN. Trail Making Test A and B: normative data stratified by age and education. Arch Clin Neuropsychol. 2004;19(2):203-214. doi:10.1016/ S0887-6177(03)00039-8

38. Salthouse TA. Selective review of cognitive aging. J Int Neuropsychol Soc. 2010;16(5):754-760. doi:10.1017/S1355617710000706
39. Shim H, Kim M, Won CW. Motoric cognitive risk syndrome is associated with processing speed and executive function, but not delayed free recall memory: The Korean frailty and aging cohort study (KFACS). Arch Gerontol Geriatr. 2020 Mar-Apr;87:103990. doi: 10.1016/j.archger.2019.103990

40. Tangney CC, Kwasny MJ, Li H, et al. Adherence to a Mediterranean-type dietary pattern and cognitive decline in a community population. Am J Clin Nutr. 2011;93(3):601-607. doi:10.3945/ajcn.110.007369

41. Ye X, Scott T, Gao X, et al. Mediterranean diet, healthy eating index 2005, and cognitive function in middle-aged and older Puerto Rican adults. J Acad Nutr Diet. 2013;113(2):276-81.e813. doi:10.1016/j.jand.2012.10.014

42. Samieri C, Sun Q, Townsend MK, et al. The association between dietary patterns at midlife and health in aging: an observational study. Ann Intern Med. 2013;159(9):584-591. doi:10.7326/0003-4819-159-9-201311050-00004

43. Durga J, van Boxtel MP, Schouten EG, et al. Effect of 3-year folic acid supplementation on cognitive function in older adults in the FACIT trial: randomised, double blind, controlled trial. Lancet. 2007;369(9557):208-216. doi:10.1016/S0140-6736(07)60109-3

44. Smith AD, Refsum H. Homocysteine, B Vitamins, and Cognitive Impairment. Annu Rev Nutr. 2016;36:211-239. doi:10.1146/ annurev-nutr-071715-050947

45. Engelhart MJ, Geerlings MI, Ruitenberg A, et al. Dietary intake of antioxidants and risk of Alzheimer disease. JAMA. 2002;287(24):3223-3229. doi:10.1001/jama.287.24.3223

46. Travica N, Ried K, Sali A, Scholey A, Hudson I, Pipingas A. Vitamin C Status and Cognitive Function: A Systematic Review. Nutrients. 2017;9(9):960. doi: $10.3390 /$ nu9090960

47. Harrison FE. A critical review of vitamin C for the prevention of age-related cognitive decline and Alzheimer's disease. J Alzheimers Dis. 2012;29(4):711726. doi:10.3233/JAD-2012-111853 